\title{
Changes in the Phenolic Concentration during Flower Development of Rose 'KORcrisett'
}

\author{
Valentina Schmitzer ${ }^{1}$, Robert Veberic, Gregor Osterc, and Franci Stampar \\ Biotechnical Faculty, Agronomy Department, Jamnikarjeva 101, SI-1000 Ljubljana, Slovenia
}

\begin{abstract}
AdDitional INDEX words. Rosa $\times$ hybrida, stages, color measurements, anthocyanins, phenolics, correlation
Abstract. The concentration of major anthocyanins, quercetins, catechin, and phenolic acids during flower development of Rosa $\times$ hybrida L. 'KORcrisett' was quantified using high-performance liquid chromatography/mass spectrometry. Additionally, the changes in petal color were monitored colorimetrically at four different stages of development (bud, partially open flowers, fully open flowers, senescent flowers) and correlation was calculated between the chromaticity parameters and major/total anthocyanins. Color parameters $a^{*}, b^{*}$, and $h^{\circ}$ decreased with the progression of flower development and $a^{*} / b^{*}$ ratio and lightness $\left(L^{*}\right)$ increased. In rose petals, a negative trend in the content of major (pelargonidin-3,5-di-O-glucoside, cyanidin-3,5-di-O-glucoside) and minor (pelargonidin-3-Oglucoside, cyanidin-3-O-glucoside, peonidin-3-O-glucoside) anthocyanins was observed during flower development. Buds contained almost threefold higher concentrations of pelargonidin-3,5-di-O-glucoside and fourfold higher concentrations of cyanidin-3,5-di-O-glucoside than senescent flowers. Buds also contained significantly more quercetins (quercetin-3-O-rutinoside, quercetin-3-O-glucoside, and quercetin-3-O-rhamnoside), catechin, and phenolic acids (gallic acid, protocatechulic acid, chlorogenic acid, caffeic acid, p-coumaric acid) than flowers of subsequent developmental stages. The most significant differences were observed in the content of gallic acid; buds contained almost sixfold higher values than senescent flowers. Correlation analysis revealed a strong correlation between chromaticity parameters $a^{*}, b^{*}, a^{*} / b^{*}$ ratio, $h^{\circ}, L^{*}$, and major/total anthocyanins with values ranging from 0.60 to $-\mathbf{0 . 8 4}$.
\end{abstract}

The popularity and commercial marketability of rose flowers primarily depends on the size, coloration, longevity, and on the scent of rose flowers. A wide spectrum of colors can be attributed to various pigments such as anthocyanins, quercetins, and carotenoids (Eugster and Markifischer, 1991) in addition to other phenolics acting as copigments (Davies and Mazza, 1993).

During flower bud opening and senescence, various events take place in a well-defined sequence such as cell division, cellular differentiation, shifts in membrane permeability, cell elongation, and a wide range of gene expression (Kumar et al., 2008a) in association with changes in concentration of endogenous plant growth regulators and secondary metabolites (Kumar et al., 2008a; Mayak and Halevy, 1972; Sood and Nagar, 2003). Little information is available on phenolic content of developing rose petals. Sood and Nagar (2003) observed a sharp increase during flower development from the flower bud opening to full bloom in Rosa bourboniana Desport and Rosa damascena Mill. In other plants such as Rosmarinus officinalis L., the content of phenolic diterpenes and flavones increased during flower development. However, the level of phenolic acids remained constant (Del Bańo et al., 2003).

Anthocyanin synthesis is an integral part of flower development and generally occurs at later stages of petal development (Weiss, 2000). The rapid petal growth, resulting from cell expansion, and anthocyanin accumulation in petals seem to be regulated by the same developmental signals (Weiss, 2000). The concentration of total anthocyanins in petals declined during flower development in Rosa $\times$ hybrida 'Happiness' and 'Pink Coronet' (Ahuja et al., 1963), Petunia

Received for publication 19 June 2009. Accepted for publication 17 July 2009. This work is part of program Horticulture No. P4-0013-0481 funded by the Slovenian Research Agency (ARRS).

${ }^{1}$ Corresponding author. E-mail: valentina.schmitzer@bf.uni-lj.si. ×hybrida Hort. (Ferrante et al., 2006), and Hydrangea macrophylla Thunb. (Yoshida et al., 2008). This decline in phenolics and anthocyanins concentration at later stages of flower development may limit the role of the peroxidase/phenolics/ ascorbic acid system in antioxidant defense and make the flower more vulnerable to oxidative stress (Takahama and Oniki, 1997). Plant tissue color is easily obtained with a portable colorimeter and several studies showed that there is a strong correlation between anthocyanins in flowers/leaves and one or more chromatic parameters (Katori et al., 2002; Schmitzer et al., 2009). Therefore, changes in the anthocyanin content could be linked to visual attributes and described by tristimulus values.

Potted miniature roses have become increasingly popular over the past few decades mostly as a result of the convenient small size, constant blooming, and rapid flower development, which also make it a suitable research material. In the present investigation, we have monitored the changes in concentration of various phenolic compounds and color parameters. To our knowledge, this work provides the first report on abundance of various anthocyanins, quercetins, catechin, and phenolic acids at four developmental stages of miniature rose 'KORcrisett'. The correlations between tristimulus color measurements and major/total anthocyanins were also evaluated.

\section{Materials and Methods}

Plant material and growth conditions. 'KORcrisett' rose plants were grown in a controlled environment glass greenhouse at $27 / 22^{\circ} \mathrm{C}$ (day/night) equipped with a cooling system under natural photoperiod. The greenhouse environmental control system was set to start cooling at $27{ }^{\circ} \mathrm{C}$. Relative humidity varied from $75 \%$ to $85 \%$. Plants were irrigated daily using a flood irrigation system with a 4 -min water $\left(18{ }^{\circ} \mathrm{C}\right)$ 
supply. Flower petals from the outermost whorls were colorimetrically analyzed and harvested in 15 replicates at four developmental stages (Fig. 1): bud [B (petals closed and fully pigmented with opened sepals)], partially open flower [PO (flowers with their two to three outer whorls unfurled)], fully open flower [FO (completely unfurled petals)], and senescent flower [S (petals rolled outward, discoloration detected, flower easily abscised)]. Petals from individual flowers were harvested at each stage and subjected to further analysis.

FlOWER COLOR MEASUREMENTS. Flower color was measured by a portable colorimeter (CR-10 Chroma; Minolta, Osaka, Japan) with $\mathrm{C}$ illuminant. The colorimeter was calibrated with a white standard calibration plate before use. In the CIE $L^{*} a^{*}$ $b^{*}$ system of color representation, the $L^{*}$ value corresponds to a dark-bright scale and represents the relative lightness of colors with a range from 0 to $100(0=$ black, $100=$ white $)$. The $a^{*}$ and $b^{*}$ values extend from -60 to $60 ; a^{*}$ negative is for green and $a^{*}$ positive is for red, and $b^{*}$ negative is for blue and positive for yellow. The $a^{*} / b^{*}$ ratio was calculated for the purpose of correlation analysis with anthocyanic pigments. The hue angle $\left(h^{\circ}\right)$ is expressed in degrees from $0^{\circ}$ to $360^{\circ}\left(0^{\circ}=\right.$ red, $90^{\circ}=$ yellow, $180^{\circ}=$ green and $360^{\circ}=$ blue). Color was measured in the middle of each petal (three replicates per flower) to ensure equal measurement conditions.

EXTRACTION AND HIGH-PERFORMANCE LIQUID CHROMATOGRAPH DETERMINATION OF PHENOLIC COMPOUNDS. Petals of individual flowers were ground to a fine powder with liquid nitrogen and $2 \mathrm{~g}$ of powder extracted with $3 \mathrm{~mL}$ methanol containing 3\% (v/v) HCOOH and 1\% (w/v) 2,6-Di-tert-butyl-4methylphenol (BHT) in an ultrasonic bath for $1 \mathrm{~h}$. Treated samples were centrifuged for $7 \mathrm{~min}$ at $12,000 \mathrm{~g}_{\mathrm{n}}$. Supernatant was filtered through a polyamide filter (Chromafil AO-45/25; Macherey-Nagel, Düren, Germany) and transferred to a vial before injection in a high-performance liquid chromatograph (HPLC) system. Samples were analyzed using a Thermo Finnigan Surveyor HPLC system (Thermo Scientific, San Jose, CA) with a diode array detector at $280 \mathrm{~nm}$ (gallic acid, protocatechuic acid, catechin, chlorogenic acid, caffeic acid, p-coumaric acid), $350 \mathrm{~nm}$ (quercetins) and $530 \mathrm{~nm}$ (anthocyanins). A HPLC column (C18, $150 \times 4.6 \mathrm{~mm}$, Gemini $3 \mu$; Phenomenex, Torrance, CA) protected with a Phenomenex security guard column operated at $25{ }^{\circ} \mathrm{C}$ was used. The injection volume was $20 \mu \mathrm{L}$ and the flow rate maintained at $1 \mathrm{~mL} \cdot \mathrm{min}^{-1}$. The elution solvents were aqueous $1 \%$ formic acid (A) and $99.8 \%$ acetonitrile (B). Samples were eluted according to the linear gradient described by Marks et al. (2007): 0 to 5 $\min , 3 \%$ to $9 \% \mathrm{~B} ; 5$ to $15 \mathrm{~min}, 9 \%$ to $16 \% \mathrm{~B} ; 15$ to $45 \mathrm{~min}, 16 \%$ to $50 \% \mathrm{~B}$; 45 to $50 \mathrm{~min}, 50 \%$ isocratic; and finally washing and reconditioning of the column. The concentrations of phenolic

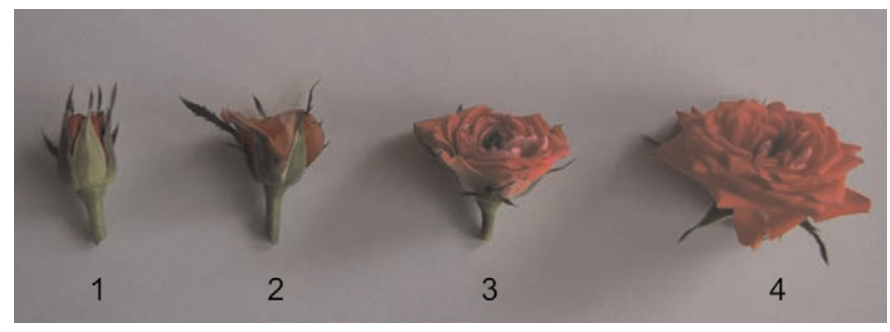

Fig. 1. Four stages of flower development: 1) bud; 2) partially open flower; 3) fully open flower; and 4) senescent flower. compounds were assessed from peak areas and quantified with the use of corresponding external standards and anthocyanins by the use of a calibration curve of cyanidin-3,5-di-O-glucoside. Anthocyanins were further identified using a mass spectrometer (LCQ Deca XP MAX; Thermo Scientific) with an electroscopy interface operating in positive ion mode using $\mathrm{MS}^{2}$ scanning mode from $\mathrm{m} / \mathrm{z} 115$ to 800 . The injection volume was $10 \mu \mathrm{L}$ and the flow rate maintained at $1 \mathrm{~mL} \cdot \mathrm{min}^{-1}$. Capillary temperature was $250{ }^{\circ} \mathrm{C}$, the sheath gas and auxiliary gas were 20 and 8 units, respectively, the capillary voltage was $26 \mathrm{~V}$, and spray voltage $4 \mathrm{~V}$. Multipole Rf amplitude was $550 \mathrm{~V}_{\mathrm{p}-\mathrm{p}}$. All compounds were expressed as $\mu \mathrm{g} \cdot \mathrm{g}^{-1}$ fresh weight (FW).

Chemicals. The standards used to determine the phenolic compounds in samples were gallic acid, (+)-catechin, quercetin3-O-rutinoside, cyanin chloride, kuromanin chloride, and chlorogenic acid from Sigma-Aldrich (Steinheim, Germany); catechin from Roth (Karlsruhe, Germany); protocatechulic acid from Merck (Darmstadt, Germany); and caffeic acid, $p$-coumaric acid, quercetin-3-glucoside, quercetin-3-O-rhamnoside, and peonidin3-O-glucoside from Fluka (Buchs, Switzerland).

The chemicals for the sample preparation and mobile phases were methanol, BHT, and acetonitrile from Sigma-Aldrich and formic acid from Fluka. The water used in the mobile phase was bidistilled and purified with a Milli-Q water purification system by Millipore (Bedford, MA).

Statistical Analysis. The results were analyzed using Statgraphics Plus 4.0 (Manugistics, Rockville, MD) program using one-way analysis of variance. Differences in phenolic concentrations among developmental stages were estimated with Duncan's multiple range test $(P<0.05)$. Multiple variable analysis with Pearson's product moment correlation coefficient $(r)$ was calculated between anthocyanins and each of the color variables at $P<0.05$.

\section{Results}

Petal color measurements. Color parameters $a^{*}, b^{*}$, and $h^{\circ}$ declined at the successive stages of flower development. However, $a^{*} / b^{*}$ ratio and lightness $\left(L^{*}\right)$ were increased. The intense red of the buds changed to pink-violet in senescent flowers (Table 1). Analysis of the color parameters $L^{*}$ and $h^{\circ}$ revealed statistically significant differences among all flower developmental stages. An increase in the parameter $L^{*}$ was observed with each change in flower stage; $L^{*}$ increased for partially opened flowers $9.25 \%$, fully opened flowers $15.60 \%$, and senescent flowers $23.52 \%$ when compared with the bud stage. On the other hand, parameter $h^{\circ}$ decreased with each change in flower stage; $h^{\circ}$ was $10.91 \%$ lower for partially opened flowers, $22.43 \%$ lower for fully opened flowers, and as much as $48.11 \%$ lower for senescent flowers when compared with the bud stage.

AnTHOCYANIN CONCENTRATION DURING FLOWER DEVELOPMENT. The HPLC chromatogram at $530 \mathrm{~nm}$ revealed different peaks corresponding to pelargonidin-3,5-di-O-glucoside $>$ cyanidin-3,5-di-O-glucoside $>$ pelargonidin-3-O-glucoside $>$ peonidin-3-O-glucoside $>$ cyanidin-3-O-glucoside. The concentration of the major anthocyanins (pelargonidin-3,5-di-O-glucoside and cyanidin-3,5-di-O-glucoside) in rose petals showed a significant negative trend during flower development. In partially opened flowers, the concentration of pelargonidin-3,5di-O-glucoside was $27.32 \%$ lower than in buds and declined to $36.36 \%$ at senescent stage (Fig. 2A). Similarly, the concentration 
Table 1. Chromatographic parameters (mean $\pm \mathrm{SE}$ ) of Rosa $\times$ hybrida 'KORcrisett' petals at four flower stages.

\begin{tabular}{|c|c|c|c|c|c|}
\hline \multirow[b]{2}{*}{ Flower stage ${ }^{z}$} & \multicolumn{5}{|c|}{ Chromatographic parameter (mean $\pm \mathrm{SE}$ ) } \\
\hline & $a^{*}$ & $b^{*}$ & $a * / b *$ ratio & $L^{*}$ & $h^{\circ}$ \\
\hline $\mathrm{B}$ & $55.49 \pm 1.66 \mathrm{~b}^{\mathrm{y}}$ & $46.21 \pm 3.60 \mathrm{c}$ & $1.40 \pm 0.16 \mathrm{a}$ & $41.08 \pm 0.72 \mathrm{a}$ & $29.33 \pm 0.51 \mathrm{~d}$ \\
\hline $\mathrm{PO}$ & $55.94 \pm 1.50 \mathrm{~b}$ & $44.42 \pm 2.69 \mathrm{c}$ & $1.44 \pm 0.18 \mathrm{a}$ & $44.88 \pm 0.69 \mathrm{~b}$ & $26.13 \pm 0.88 \mathrm{c}$ \\
\hline S & $40.96 \pm 1.88 \mathrm{a}$ & $11.30 \pm 1.14 \mathrm{a}$ & $3.56 \pm 0.26 \mathrm{c}$ & $50.74 \pm 0.73 \mathrm{~d}$ & $15.22 \pm 1.11 \mathrm{a}$ \\
\hline
\end{tabular}

${ }^{\mathrm{z}} \mathrm{B}=$ bud; $\mathrm{PO}=$ partially open flower; $\mathrm{FO}=$ fully open flower; $\mathrm{S}=$ senescent flower.

yifferent letters $(\mathrm{a}, \mathrm{b}, \mathrm{c}, \mathrm{d})$ in rows denote statistically significant differences by Duncan's multiple range test at $P<0.05$.
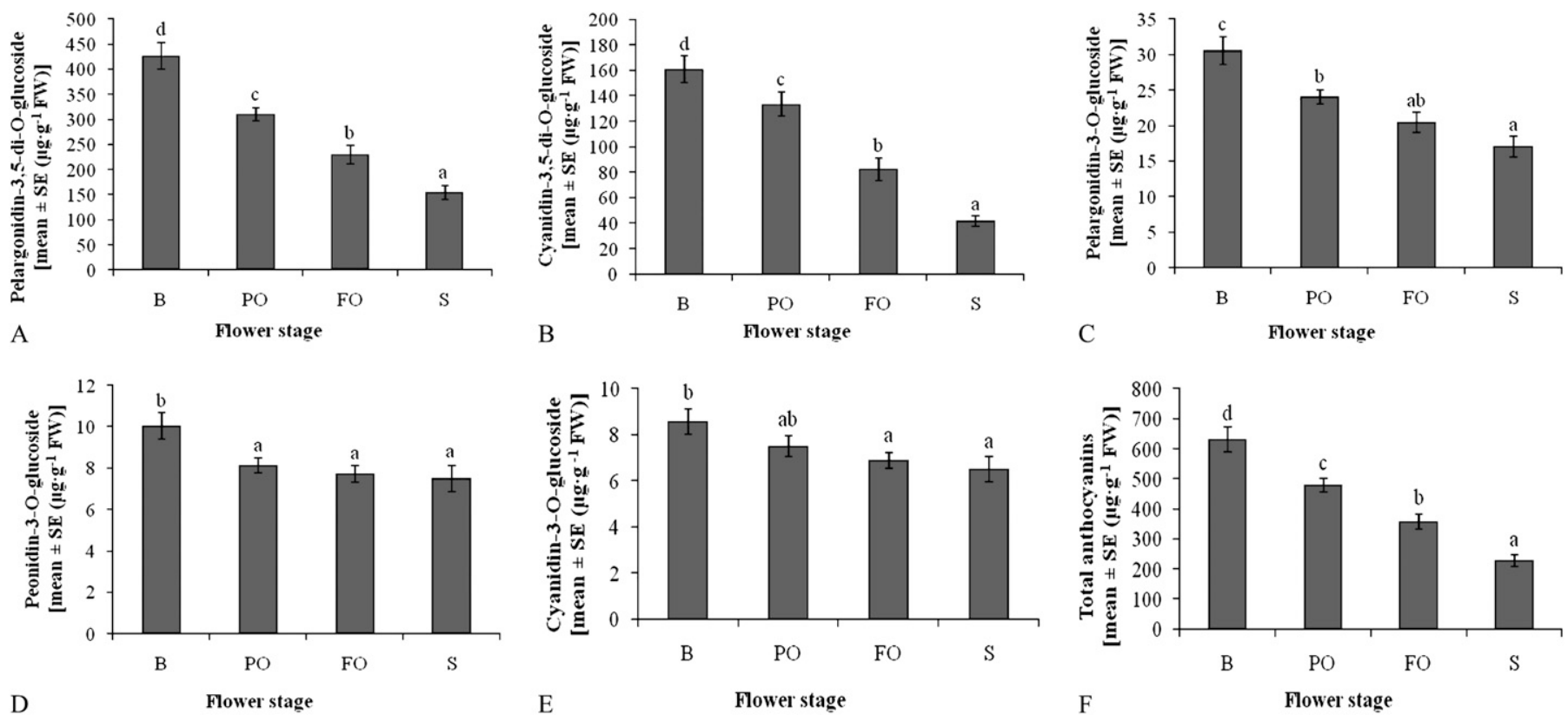

Fig. 2. Concentration of single (A-E) and total anthocyanins (F) in petals of Rosa $\times$ hybrida 'KORcrisett' at four flower stages: bud (B), partially open flower (PO), fully open flower (FO), and senescent flower (S). Different letters (a-d) denote statistically significant differences by Duncan's multiple range test at $P<0.05$.

of cyanidin-3,5-di-O-glucoside was low (26.07\%) in senescent flowers in comparison with concentrations measured in buds (Fig. 2B). A similar trend was observed in the concentration of the minor anthocyanin, pelargonidin-3-O-glucoside (Fig. 2C) from bud $\left(30.70 \pm 1.84 \mu \mathrm{g} \cdot \mathrm{g}^{-1} \mathrm{FW}\right)$ to senescent stage (17.08 \pm $\left.1.48 \mu \mathrm{g} \cdot \mathrm{g}^{-1} \mathrm{FW}\right)$. The changes in the concentration of other minor anthocyanins (cyanidin-3-O-glucoside and peonidin-3-O-glucoside) during flower development were not consistent; however, statistically significant differences were observed between the bud stage and other flower stages (Fig. 2D-E). The concentration of total anthocyanins decreased from buds to senescent flowers and statistically significant differences were observed in the concentration of total anthocyanins among all developmental stages (Fig. 2F) with values ranging from $227.70 \pm 20.47 \mu \mathrm{g} \cdot \mathrm{g}^{-1}$
FW (senescent flower) to as much $630.68 \pm 42.98 \mu \mathrm{g} \cdot \mathrm{g}^{-1}$ FW (buds).

The changes in single/total anthocyanin concentration during flower development were not accompanied by a change in the ratio among three anthocyanins (pelargonidin $>$ cyanidin $>$ peonidin) except at the senescent stage in which a smaller relative concentration of cyanidin was detected as a result of an increase in the relative value of both pelargonidins and peonidin.

CORRELATION BETWEEN ANTHOCYANINS AND COLOR MEASUREMENTS. Strong correlations were observed $(\alpha<$ 0.001 ) among major/total anthocyanins, $a^{*}, b^{*}, a^{*} / b^{*}$ ratio, $h^{\circ}$, and $L^{*}$ in rose petals (Table 2). The strongest correlation was obtained between $L^{*}$ and cyanidin-3,5-di-O-glucoside $(-0.84)$ followed by pelargonidin-3,5-di-O-glucoside $(-0.83)$

Table 2. Pearson's correlation coefficients between chromaticity parameters and major/total anthocyanins in Rosa $\times$ hybrida 'KORcrisett' flowers.

\begin{tabular}{|c|c|c|c|c|c|}
\hline \multirow[b]{2}{*}{ Anthocyanin } & \multicolumn{5}{|c|}{ Chromatographic parameter } \\
\hline & $a^{*}$ & $b^{*}$ & $a * / b$ ratio & $h^{\circ}$ & $L^{*}$ \\
\hline Cyanidin-3,5-di-O-glucoside & $0.82 * * * z$ & $0.70 * * *$ & $-0.60 * *$ & $0.80 * * *$ & $-0.84 * * *$ \\
\hline Pelargonidin-3,5-di-O-glucoside & $0.72 * * *$ & $0.75 * * *$ & $-0.75 * * *$ & $0.74 * * *$ & $-0.83 * * *$ \\
\hline
\end{tabular}

zPearson's correlation coefficients between chromaticity parameters and major/total anthocyanins with statistically significance of the estimated correlation presented at $* * P<0.01$ and $* * * P<0.001$. 
and total anthocyanins $(-0.82)$. Similarly strong correlation coefficients were also observed among cyanidin-3,5-di-O-glucoside, $a^{*}(0.82)$, and $h^{\circ}(0.80)$. Lowest correlation coefficients were obtained between $a^{*} / b^{*}$ ratio, the concentration of cyanidin-3,5di-O-glucoside $(-0.60)$, and total anthocyanins $(-0.65)$.

QUERCETINS AND CATECHIN DURING FLOWER MATURATION. Quercetin-3-O-rhamnoside was the most abundant quercetin in rose petals followed by quercetin-3-O-glucoside and quercetin3-O-rutinoside (Table 3). The concentration of quercetin-3-Orhamnoside dropped significantly during flower development; in senescent flowers, the concentration was only $55.44 \%$ of the one in buds. A similar trend was observed for quercetin-3-Orutinoside with statistically significant differences observed between bud stage and other flower developmental stages. Catechin was the dominant phenolic compound detected in rose petals and its concentration declined significantly during flower development. This decline was twofold from bud (4425.44 \pm $\left.271.52 \mu \mathrm{g} \cdot \mathrm{g}^{-1} \mathrm{FW}\right)$ to senescent stage $\left(2040.90 \pm 207.74 \mu \mathrm{g} \cdot \mathrm{g}^{-1}\right.$ FW).

Phenolic aCids during flower maturation. Caffeic acid (Fig. 3D) and chlorogenic acid (Fig. 3C) were present in highest concentrations in rose petals followed by protocatechulic (Fig. 3B), p-coumaric (Fig. 3E), and gallic acid (Fig. 3A). The most significant differences were observed in the concentration of gallic acid; bud contained sixfold higher concentrations of gallic acid $\left(27.66 \pm 1.27 \mu \mathrm{g} \cdot \mathrm{g}^{-1} \mathrm{FW}\right)$ than senescent flowers $\left(4.76 \pm 0.62 \mu \mathrm{g} \cdot \mathrm{g}^{-1} \mathrm{FW}\right)$ and a clear negative trend was also observed from partially open $\left(13.91 \pm 1.15 \mu \mathrm{g} \cdot \mathrm{g}^{-1} \mathrm{FW}\right)$ to fully open flowers $\left(7.47 \pm 0.51 \mu \mathrm{g} \cdot \mathrm{g}^{-1} \mathrm{FW}\right)$. Similarly, the concentration of $p$-coumaric acid dropped from the stage bud to senescent flower by almost $80 \%$. Significant differences were observed in the content of caffeic and chlorogenic acid at various stages of flower development. Buds contained more than twofold more caffeic acid than senescent flowers and threefold more chlorogenic acid.

\section{Discussion}

Flower development in $R$. $\times$ hybrida 'KORcrisett' is accompanied by a substantial change in concentration of various phenolic compounds and petal color. As the flower developed, significant differences were observed in color parameters $a^{*}$, $b^{*}$, and $L^{*}$. The parameter $a^{*}$ is associated with red coloration in rose petals (Biolley and Jay, 1993) and during 'KORcrisett' flower development, a steady decrease in this parameter was detected. Rose flowers in the final stages of flower development became paler and this characteristic visual change seems to be associated with a gradual increase of the parameter $L^{*}$. Many research studies have reported that the major anthocyanins in plant tissues exhibit a tight correlation with colorimetric indices such as lightness $\left(L^{*}\right)$ and hue angle $\left(h^{\circ}\right)$ (Jia et al., 2008; Lancaster et al., 1997; Schmitzer et al., 2009). Correlation

Table 3. Concentration of quercetins and catechin in petals of Rosa $\times$ hybrida 'KORcrisett' at four flower stages.

\begin{tabular}{lccrr}
\hline & \multicolumn{4}{c}{ Quercetins and catechin [mean \pm SE $\left(\mu g \cdot g^{-1}\right.$ fresh weight $\left.)\right]$} \\
\cline { 2 - 5 } Flower stage $^{\mathrm{z}}$ & Quercetin-3-O-glucoside & Quercetin-3-O-rutinoside & Quercetin-3-O-rhamnoside & Catechin \\
\hline B & $33.33 \pm 1.97 \mathrm{NS}^{\mathrm{y}}$ & $11.75 \pm 1.29 \mathrm{~b}$ & $229.35 \pm 13.12 \mathrm{c}$ & $4425.44 \pm 271.52 \mathrm{c}$ \\
PO & $19.82 \pm 0.84 \mathrm{NS}$ & $6.17 \pm 0.47 \mathrm{a}$ & $135.75 \pm 5.40 \mathrm{~b}$ & $3673.41 \pm 187.88 \mathrm{~b}$ \\
FO & $16.13 \pm 1.42 \mathrm{NS}$ & $6.57 \pm 0.74 \mathrm{a}$ & $103.10 \pm 10.00 \mathrm{a}$ & $2574.03 \pm 182.59 \mathrm{a}$ \\
S & $24.24 \pm 3.11 \mathrm{NS}$ & $8.63 \pm 1.20 \mathrm{a}$ & $102.20 \pm 13.19 \mathrm{a}$ & $2040.90 \pm 207.74 \mathrm{a}$
\end{tabular}

${ }^{\mathrm{z}} \mathrm{B}=$ bud; $\mathrm{PO}=$ partially open flower; $\mathrm{FO}=$ fully open flower; $\mathrm{S}=$ senescent flower.

${ }^{y}$ Different letters in rows ( $\mathrm{a}, \mathrm{b}, \mathrm{c}, \mathrm{NS}=$ nonsignificant) denote statistically significant differences by Duncan's multiple range test at $P<0.05$.
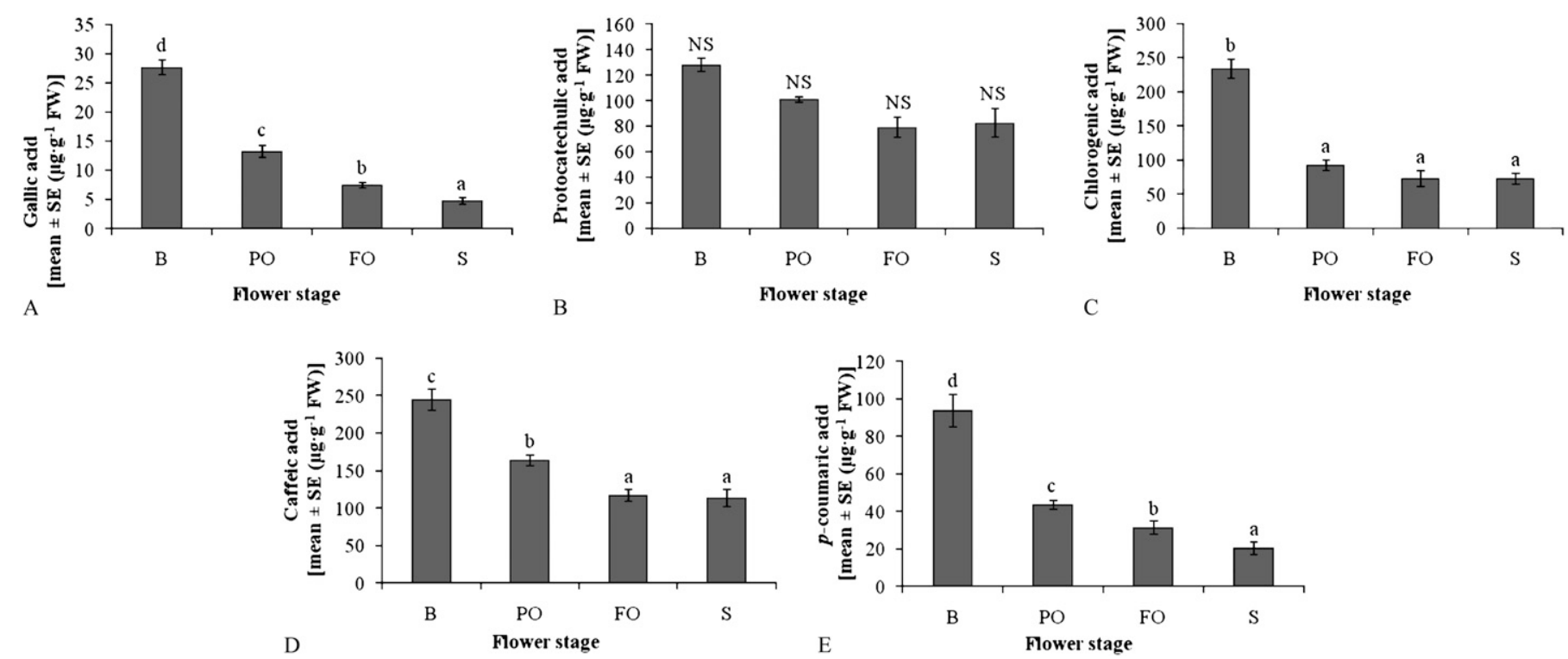

Fig. 3. Concentration of phenolic acids (A-E) in petals of Rosa $\times$ hybrida 'KORcrisett' at four flower stages: bud (B), partially open flower (PO), fully open flower (FO), and senescent flower (S). Different letters (a-d, NS = nonsignificant) denote statistically significant differences by Duncan's multiple range test at $P<0.05$. 
analysis revealed a tight correlation between tristimulus values and major/total anthocyanins in 'KORcrisett' flower petals with the best correlation obtained between major/total anthocyanins and the parameter $L^{*}$. The correlation coefficient was negative between lightness $\left(L^{*}\right)$ and the concentration of anthocyanins. Five anthocyanins detected in the petals of $R$. $\times$ hybrida 'KORcrisett' were pelargonidin-3,5-di-O-glucoside, cyanidin3,5-di-O-glucoside, pelargonidin-3-O-glucoside, peonidin-3O-glucoside, and cyanidin-3-O-glucoside. Occurrence of similar anthocyanins was also reported in other rose cultivars (Asen, 1982; Biolley et al., 1994; Mikanagi et al., 1995). The major anthocyanins in miniature rose 'KORcrisett' throughout the development were pelargonidin-3,5-di-O-glucoside and cyanidin-3,5-di-O-glucoside, which is in accordance with the results of Biolley et al. (1994) who reported that diglucosides predominate over the related 3-monoglucosides and the cooccurrence of pelargonidin and cyanidin is common in modern roses (Mikanagi et al., 1995).

Although research reports on individual anthocyanin turnover in living plant tissues are scarce, it is well evident that the concentration of single and total anthocyanins is not constant during flower development (Dela et al., 2003). In our study, the concentration of three major anthocyanins (pelargonidin-3,5di-O-glucoside, cyanidin-3,5-di-O-glucoside, pelargonidin-3O-glucoside) dropped drastically from bud to senescent stage. However, the concentration of peonidin-3-O-glucoside and cyanidin-3-O-glucoside remained constant. Several factors might be responsible for this threefold decrease in the concentration of major anthocyanins from bud to senescent stage. First, active degradation of anthocyanin was observed in Brunfelsia calycina Benth. (Vaknin et al., 2005) and $P$. $\times$ hybrida (Ferrante et al., 2006). Second, petal expansion mediated dilution of pigments and increase in flower weight (Vaknin et al., 2005). Third, it can be the result of a cessation of petal expansion at later stages of development. Probably anthocyanin concentration and the process of senescence are tightly linked phenomenon in rose flowers. Flower development in miniature rose 'KORcrisett' also revealed a significant negative trend in analyzed quercetins, catechin, and phenolic acids from bud to senescent stage. Quercetin-3-O-glucoside, quercetin-3-O-rhamnoside, and quercetin-3-O-rutinoside were the major quercetins in rose petals (Asen, 1982; Cai et al., 2005; Mikanagi et al., 1995) and their concentration declined with flower development. Gallic acid and protocatechuic acid, catechin, chlorogenic, caffeic, and $p$-coumaric acid (Cai et al., 2005; Kumar et al., 2008b; Velioglu and Mazza, 1991) are known components in rose petals and with progression of flower development in roses, the decrease in the concentration of these compounds was detected. Again, several factors might explain this gradation. Sequential biosynthesis of flavonols and anthocyanins in rose flowers could be strictly developmentally regulated like in Eustoma grandiflora (Raf.) Shinn. in which the flavonol concentration declined during floral development (Noda et al., 2004). On the other hand, it may be the result of a decline in PAL, CHI, DFR, and other gene transcriptions as was reported in Malus $\times$ domestica Borkh. flowers (Dong et al., 1998).

Flower development involves a number of interrelated processes such as growth, senescence, and abscission (Sood and Nagar, 2003) and our investigation showed a temporal decline in certain specific anthocyanic pigments and phenolics. This study provides an insight to explore the possible role of phenolics in regulation of flower development. The future work will evaluate the relative importance of these phenolics in a large number of rose cultivars for their antioxidant capacities and responses to pathogens at various stages of flower development.

\section{Literature cited}

Ahuja, K.G., H.L. Mitchell, and W.J. Carpeter. 1963. Quantitative determination of anthocyanidins from petals of rose cultivars 'Pink Coronet' and 'Happiness'. Proc. Amer. Soc. Hort. Sci. 83:829-832.

Asen, S. 1982. Identification of flavonoid chemical markers in roses and their high pressure liquid chromatographic resolution and quantification for cultivar identification. J. Amer. Soc. Hort. Sci. 107:744-750.

Biolley, J.P. and M. Jay. 1993. Anthocyanins in modern roses - Chemical and colorimetric features in relation to the color range. J. Expt. Bot. 44:1725-1734.

Biolley, J.P., M. Jay, and M.-R. Viricel. 1994. Flavonoid diversity and metabolism in 100 Rosa $\times$ hybrida cultivars. Phytochemistry 35:413-419

Cai, Y.-Z., J. Xing, M. Sun, Z.-Q. Zhan, and H. Corke. 2005. Phenolic antioxidants (hydrolysable tannins, flavonols, and anthocyanins) identified by LC-ESI-MS and MALDI-QIT-TOF MS from Rosa chinensis flowers. J. Agr. Food Chem. 53:9940-9948.

Davies, A.J. and G. Mazza. 1993. Copigmentation of simple and acylated anthocyanins with colorless phenolic compounds. J. Agr. Food Chem. 41:716-720.

Del Bańo, M.J., J. Lorente, J. Castillo, O. Benavente-García, J.A. Del Río, A. Ortuńo, K.-W. Quirin, and D. Gerard. 2003. Phenolic diterpenes, flavones, and rosmarinic acid distribution during the development of leaves, flowers, stems, and roots of Rosmarinus officinalis. Antioxidant activity. J. Agr. Food Chem. 51:4247-4253.

Dela, G., E. Or, R. Ovadia, A. Nissim-Levi, D. Weiss, and M. OrenShamir. 2003. Changes in anthocyanin concentration and composition in 'Jaguar' rose flowers due to transient high-temperature conditions. Plant Sci. 164:333-340.

Dong, Y.H., L. Beuning, K. Davies, D. Mitra, B. Morris, and A. Kootstra. 1998. Expression of pigmentation genes and photoregulation of anthocyanin biosynthesis in developing Royal Gala apple flowers. Aust. J. Plant Physiol. 25:245-252.

Eugster, C.H. and E. Markifischer. 1991. The chemistry of rose pigments. Angew. Chem. Int. Ed. Engl. 30:654-672.

Ferrante, A., P. Vernieri, F. Tognoni, and G. Serra. 2006. Changes in abscisic acid and flower pigments during flower senescence of petunia. Biol. Plant. 50:581-585.

Jia, N., Q.-Y. Shu, L.-S. Wang, H. Du, Y.-J. Xu, and Z.-A. Liu. 2008. Analysis of petal anthocyanins to investigate color mechanism in herbaceous peony cultivars. Scientia Hort. 117:167-173.

Katori, M., K. Watanabe, K. Nomura, and K. Yoneda. 2002. Cultivar differences in anthocyanin and carotenoid pigments in the petals of the flowering lotus (Nelumbo spp.). J. Jpn. Soc. Hort. Sci. 71:812-817.

Kumar, N., G.C. Srivastava, and K. Dixit. 2008a. Flower bud opening and senescence in roses (Rosa hybrida L.). Plant Growth Regulat. 55:81-99.

Kumar, N., P. Bhandari, B. Singh, A.P. Gupta, and V.K. Kaul. 2008b. Reversed phase-HPLC for rapid determination of polyphenols in flowers of rose species. J. Separation Sci. 31:262-267.

Lancaster, J.E., C.E. Lister, P.F. Reay, and C.M. Triggs. 1997. Influence of pigment composition on skin color in a wide range of fruit and vegetables. J. Amer. Soc. Hort. Sci. 122:594-598.

Marks, S.C., W. Mullen, and A. Crozier. 2007. Flavonoid and chlorogenic acid profiles of English cider apples. J. Sci. Food Agr. 87:719-728.

Mayak, S. and A.H. Halevy. 1972. Interrelationship of ethylene and abscisic acid in the control of rose petal senescence. Plant Physiol. 50:341-346.

Mikanagi, Y., M. Yokoi, Y. Ueda, and N. Saito. 1995. Flower flavonol and anthocyanin distribution in subgenus Rosa. Biochem. Syst. Ecol. 23:183-200. 
Noda, I., Y. Kanno, N. Kato, K. Kazuma, and M. Suzuki. 2004. Regulation of gene expression involved in flavonol and anthocyanin biosynthesis during petal development in lisianthus (Eustoma grandiflorum). Physiol. Plant. 122:305-313.

Schmitzer, V., G. Osterc, R. Veberic, and F. Stampar. 2009. Correlation between chromaticity values and major anthocyanins in seven Acer palmatum Thunb. cultivars. Scientia Hort. 119:442-446.

Sood, S. and P.K. Nagar. 2003. Changes in abscisic acid and phenols during flower development in two diverse species of rose. Acta Physiol. Plant. 25:411-416.

Takahama, U. and T. Oniki. 1997. A peroxidase/phenolics/ascorbate system can scavenge hydrogen peroxide in plant cells. Physiol. Plant. 101:845-852.
Vaknin, H., A. Bar-Akiva, R. Ovadia, A. Nissim-Levi, I. Forer, D. Weiss, and M. Oren-Shamir. 2005. Active anthocyanin degradation in Brunfelsia calycina (yesterday-today-tomorrow) flowers. Planta 222:19-26.

Velioglu, Y.S. and G. Mazza. 1991. Characterisation of flavonoids in petals of Rosa damascena by HPLC and spectral analysis. J. Agr. Food Chem. 39:463-467.

Weiss, D. 2000. Regulation of flower pigmentation and growth: Multiple signaling pathways control anthocyanin synthesis in expanding petals. Physiol. Plant. 110:152-157.

Yoshida, K., I. Daisuke, Y. Shinkai, and T. Kondo. 2008. Change of color and components in sepals of chameleon hydrangea during maturation and senescence. Phytochemistry 69:3159-3165. 